\title{
Nuevos programas de información y prevención en Europa para reducir los riesgos del consumo de alcohol durante el embarazo y la aparición del Síndrome Alcohólico Fetal y sus efectos relacionados
}

\section{New informative and prevention programs in Europe to reduce the risks associated to alcohol consumption during pregnancy and the appearance of Foetal Alcohol Spectrum Disorders}

I Consuelo Guerri
| Centro de Investigación Principe Felipe

Enviar correspondencia a:

Consuelo Guerri. Centro de Investigación Principe Felipe. Avda.

Autopista del Saler 16, 46012-Valencia. Tel.: 963289680

Fax: 9632897 01. E-mail: guerri@cipf.es
RESUMEN

Evidencias clínicas, epidemiológicas y experimentales durante los últimos 40 años han demostrado que el alcohol es un teratogeno y su consumo durante el embarazo puede causar muerte fetal, malformaciones, $y$, casi constantemente, alteraciones cognitivas y de la conducta en el niño. La consecuencia mas grave es el sindrome alcohólico fetal (SAF), que se observa en hijos de madres que han consumido altas cantidades de alcohol durante el embarazo. La prevalencia del SAF y de otros efectos relacionados con el consumo de alcohol se describieron en los años 1980 tanto en EEUU como en diferentes países Europeos, incluyendo España. Sin embargo a pesar de estas evidencias, muchos paises europeos han tendido a minimizar o a olvidarse de los riesgos que puede conllevar la exposición prenatal al alcohol. Treinta años más tarde, nuevos estudios clínicos así como el desarrollo de biomarcadores asociados a daño fetal por alcohol, han permitido identificar poblaciones de riesgo en Europa y posibles efectos de la exposición fetal al alcohol. En base a estos nuevos hallazgos, y coincidiendo con la Presidencia Sueca en la UE, se organizo en el Parlamento Europeo una Conferencia sobre "ALCOHOL y EMBARAZO". En este artículo se resume las diferentes ponencias y los programas de prevención e información iniciados en algunos paises de la UE. La principal conclusión de la Conferencia fue la necesidad de desarrollar programas de información en los diferentes países miembros de la UE para prevenir las consecuencias del consumo de alcohol durante la gestación.

Palabras clave: consumo de alcohol, gestación, daño fetal, síndrome alcohólico fetal.

\section{ABSTRACT}

In the last 40 years a vast mass of clinical, epidemiological and experimental evidence has demonstrated that alcohol is a teratogenic agent and that its consumption during gestation can cause foetal death, malformations and cognitive and behavioral dysfunctions in the exposed fetus. The most dramatic presentation is the complete foetal alcohol syndrome (FAS), which is observed in children born from heavy alcohol consuming mothers. FAS and of other fetal alcohol-related effects was reported in both USA and Europe in the middle 80's. However, despite these evidences, many European countries have largely forgotten or minimize the risks associated with prenatal ethanol exposure. Thirty years later, new epidemiological and clinical studies as well as new biomarkers of fetal-alcohol damage have identified high risk populations and have provided data demonstrating that significant number of women in the EU drink during pregnancy. In September of 2009, a conference on "ALCOHOL AND PREGNANCY" was organized sponsored by the Swedish Presidency of the European Union, to discuss political interventions in the EU concerning this question. I briefly summarize here the discussions, presentations and the prevention programs of some European countries. The main conclusion of the conference was that we need more information and prevention programs to improve prevention of the harmful consequences of alcohol consumption during gestation.

Key Words: alcohol consumption, gestation, fetal damage, fetal alcohol syndrome 
$\mathrm{D}$ esde que Lemoine describió en 1968 y Jones y cols. acuñaron en 1973 el término síndrome alcohólico fetal (SAF), numerosos trabajos han demostrado que el alcohol es teratógeno, pudiendo causar alteraciones en el hijo expuesto al mismo intrauterinamente que pueden resultar en muerte fetal ${ }^{2,3}$. Las alteraciones incluyen casi constantemente retrasos cognitivos y trastornos de la conducta, incluso para exposiciones a niveles relativamente bajos de alcohol. Así, el consumo de alcohol durante el embarazo es una de las principales causas prevenibles de defectos congénitos y de alteraciones de la conducta en el mundo occidental (Academia de Pediatría de Estados Unidos, 2000). ${ }^{4}$ Hoy se usa el acrónimo anglosajón FASD (Fetal Alcohol Spectrum Disorders) para englobar todo el amplio espectro de alteraciones asociadas a la exposición fetal al alcohol, ${ }_{1}^{5}$ la mayoría de carácter permanente ${ }^{6,7,8}$.

Se estima una prevalencia de SAF en el mundo occidental de $0.5-5 / 1000$ nacidos vivos ${ }^{7}$. En algunas comunidades Sudafricanas la prevalencia llega a alcanzar a 65,2$74,2 / 1,000$ nacidos vivos ${ }^{9}$. La prevalencia de niños con FASD sin dismorfia facial se ha estimado es aproximadamente 10 veces superior a la del SAF (9-10/1000) ${ }^{9}$.

Hacia los años 80 se estimó que la incidencia de SAF $(2,8 / 1000)$ y la prevalencia de los efectos del alcohol sobre el neurodesarrollo eran, en países europeos (Francia, Alemania, Suecia, Suiza) ${ }^{10}$, similares a las descritas en EEUU. En España se estimó, a partir de una muestra relativamente pequeña, que la incidencia de SAF es de 2/1000 niños nacidos vivos ${ }^{11}$. Sin embargo, a pesar de todas estas evidencias cuantitativas, se ha tendido a minimizar el problema en ciertos paises europeos, llegándose incluso a afirmar que $120 \mathrm{gr}$ de alcohol/ semana en embarazadas no llegaban a inducir daño fetal ni alteraciones en el niño ${ }^{12}$.

Ahora, 30 años más tarde, nuevos estudios clínicos y epidemiológicos ${ }^{13}$ así como el desarrollo de biomarcadores ${ }^{14}$ asociados a daño fetal por alcohol, han permitido identificar poblaciones de riesgo en Europa y posibles efectos de la exposición fetal al alcohol. Estos estudios han hecho resurgir el interés sobre este tema y han inducido al desarrollo de programas de información y prevención, que tratan de paliar o reducir los riesgos del uso (y abuso) de alcohol durante el embarazo. Algunos de estos estudios se presentaron el 9 de Septiembre de 2010 en la Conferencia Europea sobre ALCOHOL y EMBARAZO que tuvo lugar en el Parlamento Europeo.

La Conferencia fue organizada por EUROCARE (European Alcohol Policy Aliance, de la que Socidrogalcohol es miembro) y la Presidencia Sueca de la UE. A la reunión acudieron unos 100 profesionales de varias organizaciones no estatales de diferentes países miembros de la UE

La Conferencia fue inaugurada por Androulla Vassiliou (Comisionaria de Salud de la UE) y Maria Larsson (Ministra de Salud de Suecia). Ambas apoyaron el desarrollo de políticas, campañas y guías de información sobre las consecuencias del consumo materno de alcohol durante la gestación, tanto por la UE como por los paises miembros; la ayuda y el soporte técnico para abandonar la bebida a madres con problemas con el alcohol; y la prevención del consumo y abuso de alcohol en jóvenes y niños.

Diana Black (FAS Striching Fountation, Holanda) revisó brevemente los efectos del alcohol en el desarrollo fetal y presentó algunos datos sobre el impacto del consumo moderado durante la gestación en el desarrollo de alteraciones conductuales en los niños.

La magnitud de los problemas relacionados con el consumo y abuso de alcohol durante el embarazo en Europa se abordaron por 3 ponencias: 1) Prof. Mauro Ceccanti (Universita La Sapienza di Roma) mostró la alta prevalencia de niños con síndrome alcohólico fetal o SAF (3.7-7.4 /1000 niños) y con FASD (20.3-40.5 /1000 niños) en un estudio retrospectivo realizado en niños escolares de la región de Lazio (Roma) ${ }^{14}$. 2) El Dr Oriol Vall del Hospital del Mar de Barcelona, determinó en meconio ésteres etílicos de ácidos grasos (siglas en ingles, FAEEs) como nuevo biomarcador de daño fetal asociado a la exposición al alcohol, encontrando que $45 \%$ de las 353 muestras estudiadas presentaban elevados niveles de $\mathrm{FAEEs}^{13}$, sugiriendo la exposición al alcohol en una fracción alta de embarazos. 3) La Dra. Siobham Barry presentó un estudio que cubría los años 1987-2006, en el que evaluó cuántas pacientes atendidas en el Coombe Women's Hospital de Dublín durante este periodo conocían los efectos adversos del consumo de alcohol durante el embarazo. Los resultados demostraron que mientras en 1992 el 58\% de las mujeres conocian estos peligros, esta proporción descendió en 2006 a sólo el $44 \%$, de las que aproximadamente $2 / 3$ consumían alcohol durante el embarazo. De estas ultimas, 2/3 tenían $<18$ años, aunque el máximo consumo se dió en el rango de edad de 18-24 años. La conclusión de este estudio es que es urgente adoptar medidas informativas para prevenir los riesgos de esta conducta.

Otra sesión se dedicó a planes de prevención de Cataluña, Polonia y Suecia. El Dr. Colom de la Generalitat de Cataluña presentó un plan muy elaborado para el futuro inmediato, basado en programas similares ya en uso para otras adicciones. El programa polaco, ya en ejecución, presentado por Magdalena Pietrusczka (Agencia Estatal para la prevención de los efectos relacionados con el alcohol), involucra a agencias estatales y locales, medios de comunicación y Agencias de Salud, en la actividad de difusión y de educación sanitaria que es el centro de dicho programa. Se pretende extender el programa y focalizarlo en el colectivo sanitario. El programa sueco, expuesto por Catalina Zatterstrom tiene la peculiaridad de ser ejecutado principalmente por las comadronas que en Suecia Ilevan el control y seguimiento de las mujeres embarazadas. Las embarazadas identificadas como consumidoras se incorporan a un programa especial para el abandono del consumo, también aplicado por comadronas. Dicho programa parece haber tenido un grado importante de éxito en la prevención del SAF y FASD.

La experiencia francesa de etiquetar las bebidas alcohólicas con imágenes indicativas del riesgo para la embarazada (ver Figura) fue descrita por Juliette Guillemont (Institut Nacional de Prevention et d'Education pour la 
Santé). Aunque no es un programa de prevención clásico, sí se produce una alerta en las mujeres embarazadas. La presentación de Don Schenker (Alcohol Concerni, British National Agency on the Misuse of Alcohol) enfatizó los obstáculos al uso obligatorio en el Reino Unido de dicho etiquetado, por la fuerte resistencia legal y lobbying de la industria de bebidas alcohólicas. Como consecuencia el etiquetado es meramente voluntario. La última comunicación correspondió al Dr Reinhold Feldmann en el que mostró diferentes niños de su policlínica de SAF en Alemania.

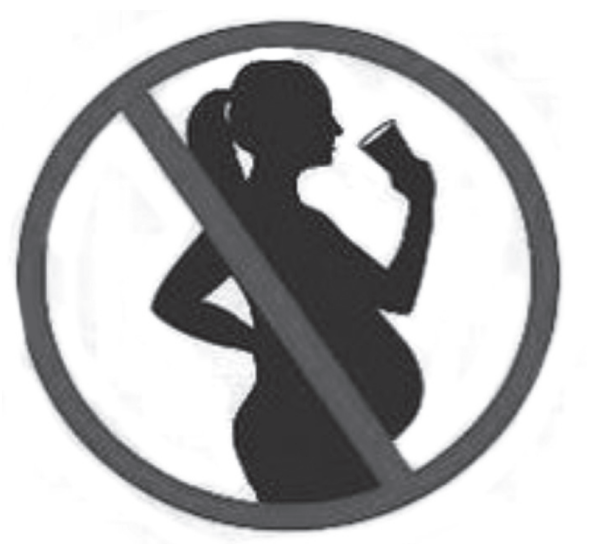

Finalmente, la recomendación unánime fue la abstinencia total durante el embarazo. Esta recomendación se basa en evidencias que indican que el consumo incluso moderado de alcohol por la madre gestante puede causar disfunciones cognitivas y alteraciones de la conducta de su hijo ${ }^{15,16}$. Así, estudios retrospectivos indican que consumos diarios de 12-24 gr etanol, es decir, una a dos copas (100-200 ml) de vino, o 1-2 cervezas de 5\% de contenido alcohólico, se asocian generalmente a alteraciones relativamente sutiles del comportamiento del hijo (falta de atención, hiperactividad, deficiencias en la resolución de problemas aritméticos, bajo rendimiento escolar, etc.) que se manifiestan en la infancia 0 en la edad escolar ${ }^{15,16,17}$. Otros estudios también demuestran que niños de 6 y 7 años de madres que consumieron una sola bebida alcohólica a la semana durante el embarazo tienen más probabilidades de presentar problemas de conducta tales como agresividad o delincuencia, que los hijos de las madres que no consumieron ${ }^{18}$.

En base a estos estudios no se puede asegurar una dosis umbral "libre de riesgos" y por tanto no existe un nivel seguro de ingesta de alcohol durante el embarazo.

\section{REFERENCIAS}

1. Jones $\mathrm{KL}$, Smith DW, Ulleland $C N$, Streissguth P. Pattern of malformation in offspring of chronic alcoholic mothers. Lancet. 1973; 1:1267-71.

2. Kesmodel U, Wisborg K, Olsen SF, Henriksen TB, Secher NJ. The rate of stillbirth increases across the amount of alcohol intake, from $1.37 / 1000$ births for human consuming $<1$ drink/week to $8.83 / 1000$ births for women consuming $\geq 5$ driks/week. Am J. Epidemiol. 2002,15;155:305-12.
3. Aliyu MH, Wilson RE, Zoorob R, Chakrabarty S, Alio AP, Kirby RS, Salihu HM .A dose-response was demonstrated, mothers who consumed five or more drinks per week during pregnancy experienced a 70\% elevated risk of stillbirth, compared with nondrinking mothers. Alcohol 2008; 42:369-74.

4. American Academy of Pediatrics. Committee on Substance Abuse and Committee on Children With Disabilities. Fetal alcohol syndrome and alcohol-related neurodevelopmental disorders. Pediatrics. 2000; 106:358-61.

5. Guerri C, Bazinet A, Riley EP. Foetal Alcohol Spectrum Disorders and alterations in brain and behaviour. Alcohol and Alcoholism 2009; 44(2):108-14.

6. Guerri C. Mechanisms involved in central nervous system dysfunctions induced by prenatal ethanol exposure. Neurotox Res. 2002; 4:327-35.

7. Guerri $C$, Rubio V. Alcohol, gestación y alteraciones infantiles. JANO 2006; 1611: 29-33

8. Sokol RJ, Delaney-Black V, Nordstrom B. Fetal alcohol spectrum disorder. JAMA 2003; 290:2996-9.

9. Bertrand J, Floyd II, Weber MK, National Center on Bird Defects and Developmental Disabilities. Guidelines for identifying and referring persons with fetal alcohol syndrome. MMWR Recomm Rep. 2005 Oct 28; 54(RR-11):1-14.

10. Sampson PD, Streissguth AP., Bookstein FL et al., Incidence of fetal alcohol syndrome and prevalence of alcohol-related neurodevelopmental disorder. Teratology 56: 317-326, 1997.

11. Romera Modamio G, Fernandez Lopez A, Jordan Garcia Y, Pastor Gomez A, Rodriguez Miguelez JM, Botet Mussons F, Figueras Aloy J. Alcoholic embryofetopathy. Neonatal case reports for the past twelve years An Esp Pediatr. 1997; 47:405-9.

12. Guerri C, Riley E, Strömland K. Commentary on the recommendations of the Royal College of Obstetricians and Gynaecologists concerning alcohol consumption in pregnancy. Alcohol Alcohol. 1999;34:497-501.

13. May PA, Fiorentino D, Phillip Gossage J, Kalberg WO, Eugene

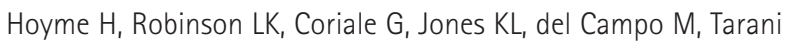
L, Romeo M, Kodituwakku PW, Deiana L, Buckley D, Ceccanti M. Epidemiology of FASD in a province in Italy: Prevalence and characteristics of children in a random sample of schools. Alcohol Clin Exp Res. 2006; 30:1562-75

14. Garcia-Algar O, Kulaga V, Gareri J, Koren G, Vall O, Zuccaro P, Pacifici R, Pichini S. Alarming prevalence of fetal alcohol exposure in a Mediterranean city. Ther Drug Monit. 2008; 30:249-54

15. Olson HC, Streissguth AP, Sampson PD, Barr HM, Bookstein $\mathrm{FL}$, Thiede K. Association of prenatal alcohol exposure with behavioral and learning problems in early adolescence.J.Am.Acad. Child.Adolesc.Psychiatry. 1997; 36:1187-94.

16. Howell KK, Lynch ME, Platzman KA, Smith GH, Coles CD. Prenatal Alcohol Exposure and Ability, Academic Achievement, and School Functioning in Adolescence: A Longitudinal Follow-Up: J Pediatr Psychol. 2006; 31:116-26.

17. Willford JA, Richardson GA, Leech SL, Day NL. Verbal and visuospatial learning and memory function in children with moderate prenatal alcohol exposure. Alcohol Clin Exp Res. 2004; 28:497-507.

18. Sood B, Delaney-Black V, Covington CH, Nordstrom-Klee B, Ager J, Templin T, Janisse J, Martier M, and Sokol R. Prenatal Alcohol Exposure and Childhood Behavior at Age 6 to 7 Years: DoseResponse Effect. PEDIATRICS $2001 ; 108,1-9$. 
